\title{
Device-free Counting via OFDM Signals of Opportunity
}

\author{
Stefania Bartoletti*, Andrea Conti*, and Moe Z. Win ${ }^{\dagger}$ \\ *DE at University of Ferrara, E-mail: stefania.bartoletti@unife.it, a.conti@ieee.org \\ ${ }^{\dagger}$ LIDS at Massachusetts Institute of Technology, E-mail: moewin@mit.edu
}

\begin{abstract}
Counting targets (people or things) within a monitored area is an important task in emerging wireless applications for smart environments, safety, and security. Counting via passive radars rely on signals of opportunity (i.e., signal already on air for other purposes) to detect and count device-free targets, which is preferable, in terms of privacy and implementation costs, to active radars that rely on dedicated or personal devices. However, conventional radar techniques for multi-target detection require to associate measurements sets to detected targets. Such data association may lead to high dimensionality and complexity even with few targets, despite it is a redundant operation for counting. The need of low dimensionality and complexity calls for the definition of signal features and the development of techniques for their extraction, which enable the association of measured signals directly with the number of targets (namely, crowd-centric algorithms). This paper develops a framework for the design and analysis of crowd-centric algorithms for device-free counting via OFDM signals of opportunity. Preliminary results in simple use cases show the effectiveness of the proposed techniques with respect to individual-centric algorithms.
\end{abstract}

Index Terms-Counting, signals of opportunity, crowd sensing, OFDM, passive radar.

\section{INTRODUCTION}

Counting targets, such as people or things, in a monitored area enables new important applications for smart environments [1], logistics [2], crowd sensing [3], public safety [4], and environmental monitoring [5]-[7]. Depending on the application and the operating environment, different approaches are considered, including image-based, devicebased, and device-free. In all of them, data are collected from multiple sensors and processed to infer the number of targets in a monitored area.

In image-based approaches, the target counting is performed by processing the foreground images collected by one or multiple cameras, after the removal of the background images [8]-[11]. In device-based approaches, the counting is performed by relying on personal or dedicated devices, such as personal smartphone or radio-frequency identification (RFID) tags [12]-[14]. Recently, device-free approaches have been investigated, where the counting is performed by sensing the wireless environment and inferring the number of targets from

This research was supported, in part, by the European Union's Horizon 2020 research and innovation programme under the Marie Skłodowska-Curie Grant 703893, the Office of Naval Research under Grants N62909-18-1-2017 and N00014-16-1-2141, and the National Science Foundation under Grant CCF-1116501. reflected signals [5], [14], [15]. Such device-free approaches preserve the privacy of the targets (no data related to the target identity) as well as they reduce the implementation cost with respect to device-based solutions. The capability of detecting and tracking people and things without relying on active devices and through the exploitation of wireless sources already covering the environment would tremendously extend the range of applications and reduce the implementation cost with maximal privacy preservation.

Device-free systems can be classified between active sensor radars (SRs) and passive SRs. Active SRs rely on a network of transmitter-receiver pairs in monostatic or multistatic configuration [16]-[19]. The signals collected at different receiving nodes are processed to detect multiple targets. Passive SRs rely on illuminators of opportunity and have been employed in the literature for stealth and low-cost tracking. In such a configuration, a network of receiving-only radars receives both the direct signal from an illuminator of opportunity and the signal backscattered by the target.

Previous works on passive radars consider VHF/UHF stations and Wi-Fi base stations as illuminators of opportunity [20]-[22]. Recently, the orthogonal frequency division multiplexing (OFDM) signals gained interest since they can be efficiently used to detect and locate targets based on Fourier analysis across subsequent blocks, which significantly reduce the computational complexity with respect to processing of other digital signals [23]-[26]. Several signal processing techniques have been proposed in the literature to detect the presence and estimate the position of a target based on the received waveforms. For example, time difference-of-arrival (TDOA), frequency difference-of-arrival (FDOA) and angleof-arrival (AOA) measurements are often adopted in this scenarios where synchronization is not guaranteed between receivers and transmitters [27]-[29].

Current solutions for multi-target detection and tracking systems rely on likelihood calculation and data association for each detected target [17], [18], [30], [31]. Data association is a computationally complex operation (growing exponentially with the number of targets) required for tracking while not necessary for counting systems that aim at estimating only the number of targets disregarding their position. Therefore, there is a growing interest in conceiving methods to count targets from the measured data without estimating the location of each single target (namely, crowd-centric methods) [15], [32], [33]. 


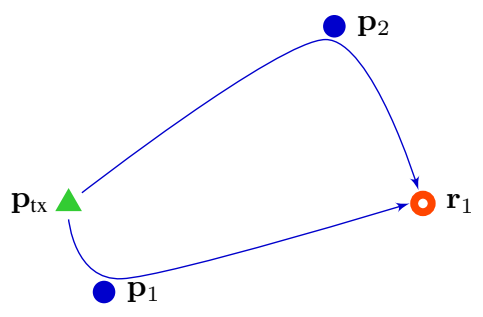

Fig. 1. Example of a portion of operating environment with one transmitter of opportunity (green triangle), one receiver (red circle) and two targets (blue circles).

This paper presents a method to count device-free targets with passive SRs without data association relying only on crowd-centric information. We propose low-complexity counting algorithms for OFDM illuminators of opportunity and based on fast Fourier transform (FFT) and OFDM channel estimation.

The remainder of the paper is organized as follows: Sec. II describes the system model; Sec. III introduces the signal processing techniques; and Sec. IV presents a case study and numerical results. Finally, in Sec. V our final remarks are given.

\section{System Model}

Consider a network of receiving-only radars with index set $\mathcal{R}$, with the $h$ th radar in position $\mathbf{r}_{h}(h \in \mathcal{R})$, monitoring an area illuminated by an OFDM transmitter at $\mathbf{p}_{\mathrm{tx}}$. Fig. 1 shows an example of the operating environment with one transmitter of opportunity, one receiver, two target scatterers and two clutter scatterers. The OFDM transmitter emits a broadcast signal at center frequency $f_{\mathrm{c}}$ with equivalent low-pass version

$$
s(t)=\sum_{i=-\infty}^{+\infty} s_{i}\left(t-i T^{\prime}\right)
$$

where, for even number $N$ of subcarriers,

$$
s_{i}(t)=\sum_{n=-N / 2}^{N / 2-1} a_{i}[n] e^{j 2 \pi n \Delta_{\mathrm{f}} t} \mathbb{1}_{\left(-T_{\mathrm{cp}}, T\right]}\{t\}
$$

in which $a_{\mathrm{i}}[n]$ is the $i$ th data symbol on the $n$th subcarrier, $\Delta_{\mathrm{f}}$ is the frequency spacing between two adjacent subcarriers, $T^{\prime}=T+T_{\mathrm{cp}}$, and $T_{\mathrm{cp}}$ is the cyclic prefix time. The transmitted signal $s(t)$ is decoded and reconstructed as $\hat{s}(t)$, for example based on the signal collected at a reference receiver (i.e., a reference signal).

Each radar receives the signal after backscattering by all the objects that are present in the operating environment, also referred to as scatterers. The dynamic scatterers (velocity and Doppler shift different than zero) are targets to be counted; the static scatterers (velocity and Doppler shift equal to zero) are present also in the absence of the targets, namely the clutter.
Therefore, the signal collected by the $h$ th radar after multipath propagation is [17]

$$
\mathrm{r}^{(h)}(t)=\mathrm{r}_{\mathrm{b}}^{(h)}(t)+\mathrm{r}_{\mathrm{t}}^{(h)}(t)+\mathrm{n}^{(h)}(t)
$$

where: $r_{\mathrm{b}}^{(h)}(t)$ is the signal component related to the background environment due to clutter and direct signal (the same component would be received in the absence of targets, when the area is empty); $r_{\mathrm{t}}^{(h)}(t)$ is the signal component related to backscattering from the targets; and $n(t)$ is the noise component. ${ }^{1}$. An estimate of the background component can be removed from the received waveform leading to

$$
\breve{\mathrm{r}}^{(h)}(t)=\mathrm{r}^{(h)}(t)-\hat{r}_{\mathrm{b}}^{(h)}(t)
$$

which is the received signal after background removal.

The residual of the background component after background removal depends on the clutter mitigation algorithm, whose analysis is beyond the scope of this paper. Experimental results show that the use of techniques that mitigate the effect of the direct signal and clutter, such as null steering, can attenuate the static background component significantly (even up to $100 \mathrm{~dB}$ ) and reduce the receiver dynamic range [23], [34]. After ideal clutter removal, the $h$ th received signal becomes

$$
\breve{r}^{(h)}(t)=\sum_{k \in \mathcal{K}^{(h)}} \alpha_{k}^{(h)} e^{j v_{k} \psi_{k}^{(h)}} \breve{s}\left(t-\tau_{k}^{(h)}\right)+\mathbf{n}^{(h)}(t)
$$

where: $\mathcal{K}^{(h)}$ is the index set of multipath components due to all the targets; $\alpha_{k}^{(h)}$ is the amplitude and $\tau_{k}^{(h)}$ is the arrival time for the $k$ th path component. In particular, $\tau_{k}^{(h)}=\left(\| \mathbf{p}_{\mathrm{tx}}-\right.$ $\left.\mathbf{p}_{\mathrm{t}}^{(k)}\|+\| \mathbf{p}_{\mathrm{t}}^{(k)}-\mathbf{r}_{h} \|\right) / c$ is the arrival time of the component backscattered by the $k$ th target at $\mathbf{p}_{\mathrm{t}}^{(k)}$ that propagates over the transmitter- $k$ th target link and the $k$ th target- $h$ th receiver link. ${ }^{2}$ For the target scatterers, $\psi_{k}^{(h)}=2 \pi \beta_{k}^{(h)} f_{\mathrm{c}} t$ where $\beta_{k}^{(h)}=f_{k}^{(h)} /\left(v f_{\mathrm{c}}\right)=\left(\cos \omega_{\mathrm{t}, k}+\cos \omega_{\mathrm{r}, k}\right) / c$ where $f_{k}^{(h)}$ is the Doppler shift, which is considered constant over a block of duration $T^{\prime}, \omega_{\mathrm{t}, k}$ is the angle describing the relative direction between the transmitter and the target, and $\omega_{\mathrm{r}, k}$ is the angle describing the relative direction between the target and the $k$ th receiver. $^{3}$

The aim of a counting system is to estimate the number of target scatterers $n_{\mathrm{t}}=\left|\mathcal{K}_{\mathrm{t}}^{(h)}\right|$ by processing the received signals $\breve{r}^{(h)}(t) \forall h \in \mathcal{R}$. The vector

$$
\breve{\mathbf{r}}=\left[\breve{\mathbf{r}}^{(1)}, \breve{\mathbf{r}}^{(2)}, \ldots, \breve{\mathbf{r}}^{(|\mathbf{r}|)}\right]
$$

\footnotetext{
${ }^{1}$ The noise samples are zero-mean Gaussian random variable (RV) $\mathbf{n}_{j}^{(h)}=$ $\mathrm{n}^{(h)}\left(t_{j}\right) \sim \mathcal{N}\left(0, \sigma_{\mathrm{n}}^{2}\right)$ at time $t_{j}$ and the variance $\sigma_{\mathrm{n}}^{2}$ is considered known to simplify the notation

${ }^{2}$ The symbol $c$ denotes the speed-of-light and $\|\cdot\|$ denotes the Euclidean distance.

${ }^{3}$ The geometry-based single-bounce model is employed, where the number of multipath components is equal to the number of scatterers. This is a widely adopted assumption for two reasons: (1) the power related to a double or multiple bounce path is proportional to the product of the radar cross section of two or multiple targets, which is negligible; and (2) this is equivalent to consider a lower spatial resolution, which is a common assumption due to bandwidth limitations that are intrinsic to the hardware that is involved [35]
} 
represents the concatenation of the vectors of received signal samples for each receiver. The length of the vector is $n_{\mathrm{m}}=$ $|\mathcal{R}| n_{\mathrm{s}}$, where $n_{\mathrm{s}}$ is the number of received signal samples $\breve{\mathrm{r}}^{(h)}\left(n T_{\mathrm{s}}\right)$ with $n=0,1, \ldots, n_{\mathrm{s}}-1$, which depends on the sampling time $T_{\mathrm{s}}$ and the observation interval $T_{\mathrm{obs}}=n_{\mathrm{s}} T_{\mathrm{s}}$. The vector

$$
\mathbf{p}_{\mathrm{t}}=\left[\mathbf{p}_{\mathrm{t}}^{(1)}, \mathbf{p}_{\mathrm{t}}^{(2)}, \ldots, \mathbf{p}_{\mathrm{t}}^{\left(n_{\mathrm{t}}\right)}\right]
$$

is the concatenation of the target position vectors. From (3) and (5), when the background is perfectly removed, i.e. $\hat{r}_{\mathrm{b}}^{(h)}(t)=$ $r_{\mathrm{b}}^{(h)}(t)$, it follows that for a given channel instantiation, $\breve{\mathbf{r}}^{(h)}$ is a random vector that depends on a deterministic and unknown parameter vector $\boldsymbol{\theta}^{(h)}=\left[\mathbf{p}_{\mathbf{t}}, \boldsymbol{\alpha}^{(h)}, \boldsymbol{\tau}^{(h)}, \mathbf{v}\right]$, where

$$
\begin{aligned}
\boldsymbol{v} & =\left[v_{1}, v_{2}, \ldots, v_{n_{\mathrm{t}}}\right] \\
\boldsymbol{\tau}^{(h)} & =\left[\tau_{1}^{(h)}, \tau_{2}^{(h)}, \ldots, \tau_{n_{\mathrm{t}}}^{(h)}\right] \\
\boldsymbol{\alpha}^{(h)} & =\left[\alpha_{1}^{(h)}, \alpha_{2}^{(h)}, \ldots, \alpha_{n_{\mathrm{t}}}^{(h)}\right] .
\end{aligned}
$$

and on the noise component $\mathbf{n}^{(h)}(t)$. The parameters $\boldsymbol{\alpha}^{(h)}$ and $\tau^{(h)}$, i.e. the arrival time and amplitude of the multipath components, depend on the channel instantiation.

\section{Signal Processing for Counting}

The proposed method relies on a matched filter receiver, i.e. a bank of correlators tuned to the transmitted waveform given a certain Doppler shift and delay. The number of correlators drives the accuracy and complexity of the counting algorithm. At the $h$ th receiver, each correlator will produce for a delay $\tau$ and Doppler shift $\phi$

$$
\mathrm{z}^{(h)}(\tau, \phi)=\int_{0}^{T_{\mathrm{obs}}} \breve{\mathrm{r}}^{(h)}(t) e^{-j 2 \pi \phi f_{\mathrm{c}}} s^{*}(t-\tau) d t
$$

where $T_{\mathrm{obs}}$ is the observation time. By considering $T_{\mathrm{obs}}$ as an integer multiple of $T^{\prime}$ and given the OFDM structure of the signal, we have

$$
\mathrm{z}^{(h)}(\tau, \phi)=\sum_{i=0}^{T_{i} / T^{\prime}} e^{-j \pi \phi f_{\mathrm{c} T}} \sum_{n=-\frac{N}{2}}^{\frac{N}{2}} \mathrm{~h}_{i, n}^{(h)} e^{j 2 \pi n \Delta_{f} \tau} .
$$

If the phase rotation can be approximated as constant within one OFDM block (the product between the Doppler shift and $T^{\prime}$ is much smaller than unity), then [23]

$$
\begin{aligned}
& \mathrm{h}_{i, n}^{(h)}=a_{i}^{*}[n] \int_{0}^{T} e^{-j 2 \pi n \Delta_{f} t_{\mathrm{r}^{(}}(h)}\left(t+i T^{\prime}\right) d t \\
& \simeq \sum_{k \in \mathcal{K}^{(h)}} \alpha_{k}^{(h)} T\left|a_{i}[n]\right|^{2} e^{j 2 \pi\left(i v_{k} \psi_{k}^{(h)} f_{\mathrm{c}} T^{\prime}-n \Delta_{f} \tau_{k}^{(h)}\right)} .
\end{aligned}
$$

At each receiver, the correlation is calculated for a finite number of tuples $\left(\tau_{j}, \phi_{j}\right)$ with $j \in \mathcal{M}=1,2, \ldots, n_{\mathrm{m}}$, which represent potential target locations and velocities. In particular, we define $T_{\mathrm{d}}$ as the temporal distance between any two pairs of tentative delays $T_{\mathrm{d}}=\tau_{j}-\tau_{i} \forall i, j=1,2, \ldots, n_{\mathrm{m}}$ and $F_{\mathrm{d}}$ as the frequentcy distance between any two pairs of tentative Doppler shifts $F_{\mathrm{d}}=\left(\phi_{j}-\phi_{i}\right) \forall i, j=1,2, \ldots, n_{\mathrm{m}}$. Therefore, $T_{\mathrm{d}}$ and $F_{\mathrm{d}}$ represent the time and frequency resolution of the receiver. The signal component backscattered from a target at the tentative position $\tilde{\boldsymbol{p}}_{j}$ such that $\tau_{j}=\left(\left\|\mathbf{p}_{\mathrm{tx}}-\tilde{\boldsymbol{p}}_{j}\right\|+\| \tilde{\boldsymbol{p}}_{j}-\right.$ $\left.\mathbf{r}_{h} \|\right) / c$ with a Doppler shift $\phi_{j}$, will contribute to the received energy at the $j$ th correlator output $z^{(h)}\left(\tau_{j}, \phi_{j}\right)$. The output of all the correlators at the $h$ th receiver is a vector $\mathbf{e}^{(h)}$ of $n_{\mathrm{m}}$ energy samples corresponding to the tentative tuples of target locations and velocity. The $j$ th energy sample is given by

$$
\begin{aligned}
\mathrm{e}_{j}^{(h)}=z^{(h)}\left(\tau_{j}, \phi_{j}\right) & \simeq \sum_{k \in \mathrm{K}_{\mathrm{t}}^{(h)}} \alpha_{k}^{(h)} T \sum_{i=0}^{T_{i} / T^{\prime}} \sum_{n=-\frac{N}{2}}^{\frac{N}{2}}\left|a_{i}[n]\right|^{2} \\
& \times e^{j 2 \pi\left(i\left(v_{k} \psi_{k}^{(h)}-\phi_{j}\right) f_{\mathrm{c}} T^{\prime}-n \Delta_{f}\left(\tau_{k}^{(h)}-\tau_{j}\right)\right)}
\end{aligned}
$$

which represents the energy collected for the $j$ th tuple $\left(\tau_{j}, \phi_{j}\right)$. Such an energy has maximum value when $\phi_{j}=v_{k} \psi_{k}^{(h)}$ and $\tau_{j}=\tau_{k}^{(h)}$ for any $k$.

The estimated number of targets $\hat{n}_{\mathrm{t}}^{(h)}$ is obtained at the $h$ th receiver from $\mathbf{e}^{(h)}$. The final estimate for the number of targets is the mean value of the number estimated by the different sensors

$$
\hat{n}_{\mathrm{t}}=\frac{1}{n_{\mathrm{s}}} \sum_{h \in \mathcal{R}} \hat{n}_{\mathrm{t}}^{(h)} .
$$

To calculate $\hat{n}_{\mathrm{t}}^{(h)}$, we propose two possible algorithms, a maximum bin search (MBS) and threshold crossing search (TCS). For both the algorithms, a threshold for each energy sample is defined by the vector $\boldsymbol{\xi}=\left[\xi_{1}, \xi_{2}, \ldots, \xi_{N_{\mathrm{m}}}\right]$. The estimated number of targets is initialized for each sensor as $\hat{n}_{\mathrm{t}, 0}^{(h)}=0$, and the energy vector is initialized as $\tilde{\mathbf{e}}_{0}=$ $\left[\mathrm{e}_{1,0}^{(h)}, \mathrm{e}_{2,0}^{(h)}, \ldots, \mathrm{e}_{n_{\mathrm{m}}, 0}^{(h)}\right]$. At the $k$ th step, the number of targets is estimated from $\tilde{\mathbf{e}}_{k}$ and $\tilde{\mathbf{e}}_{k}$ is updated through the path loss law, which is known or learned through measurements.

In particular, given the path loss law, a value $\alpha_{j}$ can be associated to the tentative arrival time $\tau_{j}$ as

$$
\alpha_{j}=Q_{j}^{(h)} \exp \left(\tau_{j} / \gamma\right)
$$

where

$$
Q_{k}^{(i)}=\frac{10^{P_{j}^{(h)}} / 10}{\sum_{k=1}^{n_{\mathrm{t}}} \tau_{j} / \gamma}
$$

$\gamma$ is the power decay constant, and $P_{j}^{(h)}$ is the received signal strength (RSS) for a target at $\tilde{\boldsymbol{p}}_{j}$ with respect to the $h$ th receiver, i.e., considering the path-loss and the radar cross section (RCS) of the corresponding scatterer [17]. ${ }^{4}$

The value $\alpha_{j}$ can be used to calculate the expected value of $\mathrm{e}_{j}^{(h)}$ when a target is at $\tilde{\boldsymbol{p}}_{j}$. If one or multiple targets are detected at $\tilde{\boldsymbol{p}}_{j}$, its energy contribution is removed from the

\footnotetext{
${ }^{4}$ The RCS measures the power density that the object reflects with respect to the incident power, in relation to scatterer orientation, material, and size [18], [36], [37].
} 

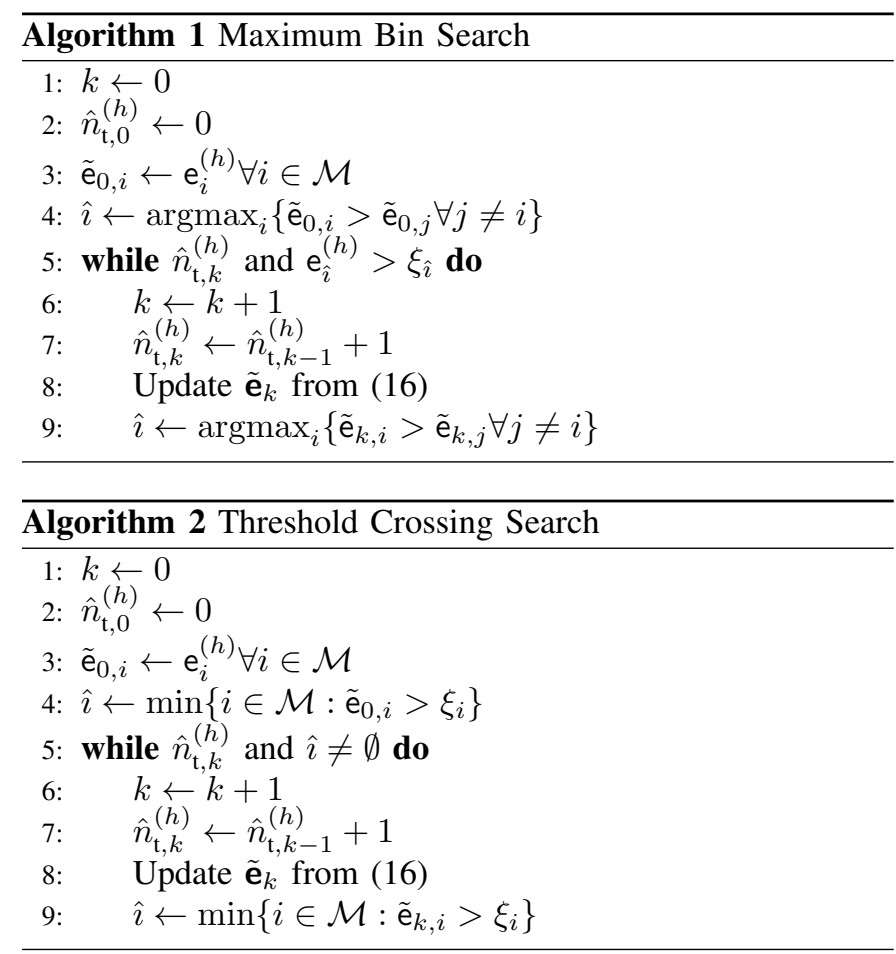

energy vector starting from $\alpha_{j}$ as

$$
\begin{aligned}
\tilde{\mathbf{e}}_{k}=\tilde{\mathbf{e}}_{k-1} & -\tilde{\alpha}_{\hat{\imath}} T \sum_{i=0}^{T_{i} / T^{\prime}} \sum_{n=-\frac{N}{2}}^{\frac{N}{2}}\left|a_{i}[n]\right|^{2} \\
& \times e^{j 2 \pi\left(i\left(\phi_{\hat{\imath}}-\phi_{j}\right) f_{\mathrm{c}} T^{\prime}-n \Delta_{f}\left(\tau_{\hat{\imath}}-\tau_{j}\right)\right)} .
\end{aligned}
$$

The MBS is presented in Alg. 1. At each iteration, the MBS algorithm first searches for the maximum value among all the bins with index $j \in \mathcal{M}$. If such maximum value is above the corresponding threshold, the number of targets is updated, the energy vector is updated by considering the , and the search is made again. The algorithms is terminated when the energy bins are all below the threshold or a maximum number of detected targets $n_{\max }$ is reached.

The TCS is presented in Alg. 2. At each iteration, the TCS algorithm searches for the first value among all the bins with index $j \in \mathcal{M}$ that overcomes the corresponding threshold. If such maximum value is above the corresponding threshold, the number of targets is updated, the energy vector is updated, and the search is made again. The algorithms is terminated when the energy bins are all below the threshold or a maximum number of detected targets $n_{\max }$ is reached.

\section{CASE Study}

Consider a squared monitored environment of $10 \mathrm{~m} \times 10 \mathrm{~m}$ with one transmitter in the center $\boldsymbol{p}_{\mathrm{tx}}=[5,5] \mathrm{m}$ and four receivers at the corners, $\boldsymbol{r}_{1}=[0,0], \boldsymbol{r}_{2}=[0,10], \boldsymbol{r}_{3}=$ $[10,10]$, and $\boldsymbol{r}_{4}=[10,0]$, with $n_{\mathrm{s}}=4$. The maximum number of targets is $n_{\max }=10$ and results are obtained via Monte Carlo simulation by varying the number of targets $n_{\mathrm{t}}=\left\{0,1,2, \ldots, n_{\max }\right\}$ and considering the targets uniformly

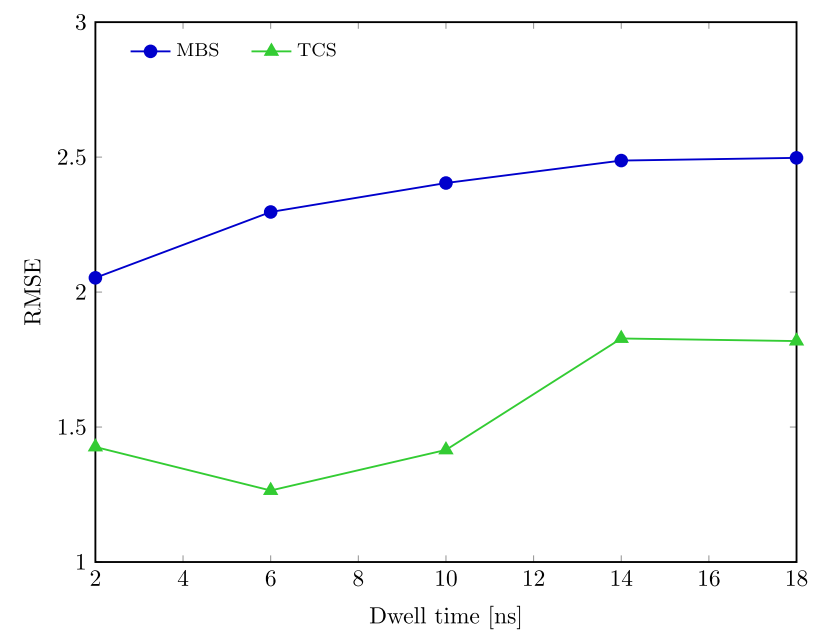

Fig. 2. RMSE for different values of $T_{\mathrm{d}}$.

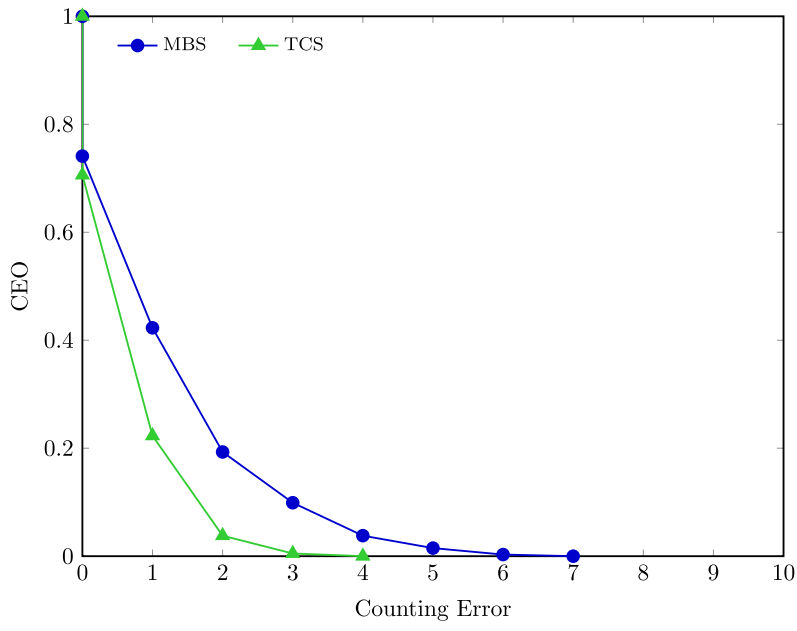

Fig. 3. Counting error outage for the MBS and TCS algorithms.

distributed in the monitored environment. The transmitted signal is a Wi-Fi signal, with bandwidth $B=20 \mathrm{MHz}$, number of carriers $N=52$, carrier spacing $\Delta_{\mathrm{f}}=312.5 \mathrm{KHz}$ according to the IEEE 802.11 standard. The symbols are perfectly reconstructed and ideal clutter removal is considered.

Counting is performed with the MBS and TCS algorithms. The performance is evaluated in terms of counting error outage $P_{\text {ceo }}(n)$, i.e. the percentage of times that the counting error $\left|\hat{n}_{\mathrm{t}}-n_{\mathrm{t}}\right|$ is above $n$ and root-mean-square of the counting error (RMSE). The resolution of the energy vector varies, with $T_{\mathrm{d}}=2,4, \ldots, 18 \mathrm{~ns}$, whereas the target velocity is assumed as known. The threshold vector is chosen as the one the minimizes the RMSE.

Fig. 2 shows the RMSE for different values of $T_{\mathrm{d}}$. It can be observed that the TCS outperforms the MBS for all the values of $T_{\mathrm{d}}$ considered. For example, for the same value of $T_{\mathrm{d}}=6 \mathrm{~ns}$, the root-mean-square error (RMSE) is 2.05 with MBS and 1.42 with TCS. Furthermore, the RMSE increases with $T_{\mathrm{d}}$ for the MBS case, and goes from 2.05 with $T_{\mathrm{d}}=2 \mathrm{~ns}$ to 2.50 with $T_{\mathrm{d}}=18 \mathrm{~ns}$, whereas, it has a minimum value 1.26 
with $T_{\mathrm{d}}=6 \mathrm{~ns}$ for the TCS case. Therefore, the time resolution has a important impact on the counting performance and its optimal value depends on the algorithm employed.

Fig. 3 shows the counting error outage $P_{\text {ceo }}(n)$ as a function of $n$ for the TCS and MBS algorithms. Results are obtained with $T_{\mathrm{d}}=2 \mathrm{~ns}$ in the MBS case and with $T_{\mathrm{d}}=6 \mathrm{~ns}$ for the MBS case, which represent the best cases in terms of RMSE according to Fig. 2. It can be observed that the MBS is outperformed by the TCS. In particular, the probability that the counting error is above 2 is 0.19 for the MBS and 0.04 for the TCS.

\section{FinAL REMARK}

A device-free counting system that relies on OFDM signals of opportunity is presented. The proposed counting system does not require data association and is based on crowd counting algorithms. The counting system performance is evaluated in a simple case study with Wi-Fi signals varying the number of targets and considering two different algorithms based on maximum search and threshold crossing. Results show the importance of the choice of time resolution for the counting performance.

\section{REFERENCES}

[1] G. Cardone, P. Bellavista, A. Corradi, C. Borcea, M. Talasila, and R. Curtmola, "Fostering participaction in smart cities: A geo-social crowdsensing platform," IEEE Commun. Mag., vol. 51, no. 6, pp. 112 119, Jun. 2013.

[2] X. Wang, S. Yuan, R. Laur, and W. Lang, "Dynamic localization based on spatial reasoning with RSSI in wireless sensor networks for transport logistics," Sensors and Actuators A: Physical, vol. 171, no. 2, pp. 421428, Nov. 2011

[3] F. Zabini and A. Conti, "Inhomogeneous Poisson sampling of finiteenergy signals with uncertainties in $\mathbb{R}^{d}, "$ IEEE Trans. Signal Process., vol. 64, no. 18, pp. 4679-4694, Sep. 2016.

[4] S. M. George, W. Zhou, H. Chenji, M. Won, Y. O. Lee, A. Pazarloglou, R. Stoleru, and P. Barooah, "Distressnet: A wireless Ad Hoc and sensor network architecture for situation management in disaster response," IEEE Commun. Mag., vol. 48, no. 3, pp. 128-136, Mar. 2010.

[5] E. Cianca, M. D. Sanctis, and S. D. Domenico, "Radios as sensors," IEEE Internet of Things Journal, 2016, to appear.

[6] A. Basalamah, "Sensing the crowds using bluetooth low energy tags," IEEE Access, vol. 4, pp. 4225-4233, Aug. 2016.

[7] S. Savazzi, S. Sigg, M. Nicoli, V. Rampa, S. Kianoush, and U. Spagnolini, "Device-free radio vision for assisted living: Leveraging wireless channel quality information for human sensing," vol. 33, no. 2, pp. 4558, Mar. 2016.

[8] Y. L. Hou and G. K. H. Pang, "People counting and human detection in a challenging situation," IEEE Transactions on Systems, Man, and Cybernetics - Part A: Systems and Humans, vol. 41, no. 1, pp. 24-33, Jan 2011.

[9] N. C. Tang, Y. Y. Lin, M. F. Weng, and H. Y. M. Liao, "Cross-camera knowledge transfer for multiview people counting," IEEE Transactions on Image Processing, vol. 24, no. 1, pp. 80-93, Jan 2015.

[10] B. K. Dan, Y. S. Kim, Suryanto, J. Y. Jung, and S. J. Ko, "Robust people counting system based on sensor fusion," IEEE Transactions on Consumer Electronics, vol. 58, no. 3, pp. 1013-1021, August 2012

[11] A. B. Chan and N. Vasconcelos, "Counting people with low-level features and Bayesian regression," IEEE Transactions on Image Processing, vol. 21, no. 4, pp. 2160-2177, April 2012.

[12] F. Guidi, N. Decarli, S. Bartoletti, A. Conti, and D. Dardari, "Detection of multiple tags based on impulsive backscattered signals," vol. 62, no. 11, pp. 3918-3930, Nov. 2014.

[13] E. Vattapparamban, B. S. Ciftler, I. Guvenc, K. Akkaya, and A. Kadri, "Indoor occupancy tracking in smart buildings using passive sniffing of probe requests," in 2016 IEEE International Conference on Communications Workshops (ICC), May 2016, pp. 38-44.
[14] S. Depatla, A. Muralidharan, and Y. Mostofi, "Occupancy estimation using only WiFi power measurements," IEEE Journal on Selected Areas in Communications, vol. 33, no. 7, pp. 1381-1393, July 2015.

[15] X. Quan, J. W. Choi, and S. H. Cho, "In-bound/out-bound detection of people's movements using an IR-UWB radar system," in Int. Conf. on Electronics, Information and Communications (ICEIC), Kota Kinabalu, Malaysia, Jan. 2014, pp. 1-2.

[16] M. I. Skolnik, "An analysis of bistatic radar," vol. ANE-8, no. 1, pp. 19-27, Mar. 1961.

[17] — Radar Handbook, 2nd ed. McGraw-Hill Professional, Jan. 1990.

[18] V. S. Chernyak, Fundamentals of Multisite Radar Systems: Multistatic Radars and Multiradar Systems, 1st ed. New York: CRC Press, Sep. 1998.

[19] E. Paolini, A. Giorgetti, M. Chiani, R. Minutolo, and M. Montanari, "Localization capability of cooperative anti-intruder radar systems," EURASIP Journal on Advances in Signal Processing, vol. 2008, pp. 1-14, 2008, article ID 726854, doi:10.1155/2008/726854.

[20] M. Glende, J. Heckenbach, H. Kuschel, S. Mueller, J. Schell, and C. Schumacher, "Experimental passive radar systems using digital illuminators (DAB/DVB-T)," in Proc. Int. Radar Symp, Cologne, Germany, Sep. 2007, pp. 411-417.

[21] H. Kuschel, J. Heckenbach, S. Mueller, and R. Appel, "On the potentials of passive, multistatic, low frequency radars to counter stealth and detect low flying targets," in Proc. IEEE Radar Conf., Rome, Italy, May 2008, pp. $1443-1448$

[22] C. Coleman, H. Yardley, and R. Watson, "A practical bistatic passive radar system for use with DAB and DRM illuminators," in Proc. IEEE Radar Conf., Rome, Italy, May 2008, pp. 1514-1519.

[23] C. R. Berger, B. Demissie, J. Heckenbach, P. Willett, and Z. Shengli, "Signal processing for passive radar using OFDM waveforms," vol. 4, no. 1, pp. 226-238, Feb. 2010.

[24] C. R. Berger, S. Zhou, and P. Willett, "Signal extraction using compressed sensing for passive radar with OFDM signals," in Proc. Int. Conf. Inf. Fusion (FUSION), Cologne, Germany, Jun. 2008, pp. 15141519.

[25] S. Bartoletti, A. Conti, and M. Z. Win, "Towards counting via passive radar using OFDM waveforms," in Proc. IEEE Int. Conf. Commun., Paris, France, May 2017, pp. 803-808.

[26] — " "Device-free counting via wideband signals," IEEE J. Sel. Areas Commun., vol. 35, no. 5, pp. 1163-1174, May 2017.

[27] K. C. Ho, L. Xiaoning, and L. Kovavisaruch, "Source localization using TDOA and FDOA measurements in the presence of receiver location errors: Analysis and solution," vol. 55, no. 2, pp. 684-696, Feb. 2007.

[28] M. Z. Win, A. Conti, S. Mazuelas, Y. Shen, W. M. Gifford, D. Dardari, and M. Chiani, "Network localization and navigation via cooperation," IEEE Commun. Mag., vol. 49, no. 5, pp. 56-62, May 2011.

[29] A. Conti, M. Guerra, D. Dardari, N. Decarli, and M. Z. Win, "Network experimentation for cooperative localization," IEEE J. Sel. Areas Commun., vol. 30, no. 2, pp. 467-475, Feb. 2012.

[30] F. Meyer, P. Braca, P. Willett, and F. Hlawatsch, "Tracking an unknown number of targets using multiple sensors: A belief propagation method,' in Proc. FUSION-16, Heidelberg, Germany, Jul. 2016, pp. 719-726.

[31] J. Williams and R. Lau, "Approximate evaluation of marginal association probabilities with belief propagation," IEEE Trans. Aerosp. Electron. Syst., vol. 50, no. 4, pp. 2942-2959, Oct. 2014.

[32] J. W. Choi, J. H. Kim, and S. H. Cho, "A counting algorithm for multiple objects using an IR-UWB radar system," in IEEE Int. Conf. on Net. Infrastr. and Digital Content, Beijing, China, Sep. 2012, pp. 591-595.

[33] J. He and A. Arora, "A regression-based radar-mote system for people counting," in Pervasive Computing and Communications (PerCom), 2014 IEEE International Conference on, Louis Missouri, USA, Mar. 2014, p. 95102.

[34] H. D. Griffiths and C. J. Baker, "Passive coherent location radar systems. part 1: performance prediction," IEE Proc. Radar, Sonar Navigation, vol. 152, no. 3, pp. 153-159, Jun. 2005.

[35] A. Meijerink and A. F. Molisch, "On the physical interpretation of the saleh-valenzuela model and the definition of its power delay profiles," vol. 62, no. 9, pp. 4780-4793, Sep. 2014.

[36] S. Bartoletti, A. Conti, and A. Giorgetti, "Analysis of UWB radar sensor networks," Cape Town, South Africa, May 2010, pp. 1-6.

[37] S. Bartoletti, A. Giorgetti, M. Z. Win, and A. Conti, "Blind selection of representative observations for sensor radar networks," IEEE Trans. Veh. Technol., vol. 64, no. 4, pp. 1388-1400, Apr. 2015. 